\title{
Research Paper: Effect of Using Back Brace for 8 Weeks on Electromyogrphic Activity in Women With Chrononic Back Pain During Gait
}

\author{
Farzad Farmani ${ }^{1}$ (D), Hamideh Khodaveisi ${ }^{2 *}$ iD, Fatemeh Alirezaei $^{3}$ (D)
}

1. Department of Orthotics and Prosthetics, School of Rehabilitation, Hamadan University of Medical Sciences, Hamadan, Iran 2. Department of Physical Education, Faculty of Human Sciences, Hamedan Branch, Islamic Azad University, Hamedan, Iran.

3. Department of Physical Education and Sport Sciences, Faculty of Sports Sciences, Ferdowsi University of Mashhad, Mashhad, Iran.

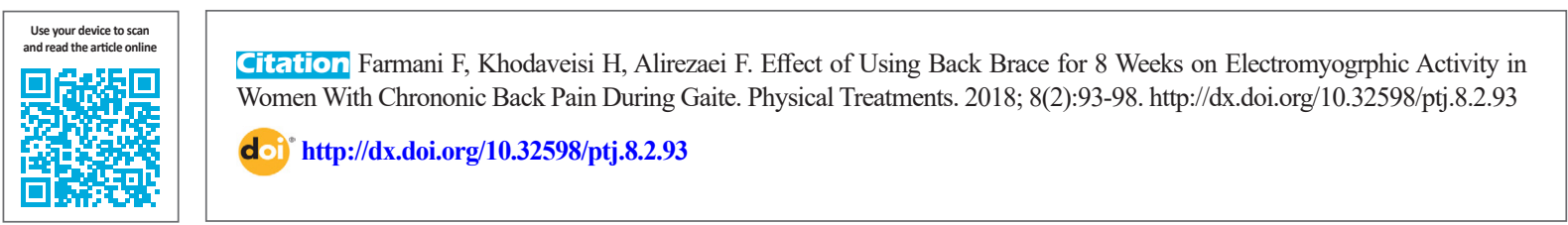

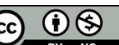

Article info:

Received: 13 Jan 2018

Accepted: 05 May 2018

Available Online: 01 Jul 2018

Keywords:

Back pain, Brace, Walking

\section{A B S T RA C T}

Purpose: The present research evaluated the effect of using a back brace for 8 weeks on the Electromyography (EMG) activity of the selected muscles among individuals with chronic back pain in the mid-stance phase of walking.

Methods: This quasi-experimental research evaluated 22 women with chronic back pain in terms of erector spinae muscles activity, abdominal erectus, abdominal external oblique, and abdominal internal oblique. The study participants were randomly selected and divided into the experimental and control groups. The experimental group employed back brace for 8 weeks. Before and after using the brace, the EMG activity was recorded in both groups. The Independent t-test and Paired t-test were used to analyze the data $(\mathrm{P} \leq 0.05)$ in SPSS.

Results: In the experimental group, the EMG activity of erector spinae and abdominal erectus muscle in the mid-stance phase of walking was significantly less than that of the control group. However, there was no significant difference in respect of external and internal oblique muscles in the experimental group compared to the controls $(\mathrm{P}>0.05)$.

Conclusion: Using brace can reduce the activity of erector spinae and abdominal erectus muscle in the mid-stance phase of walking. Kinematic and kinetic studies are necessary to describe the reasons for these changes because of back brace use.

\footnotetext{
* Corresponding Author:

Hamideh Khodaveisi, PhD.

Address: Department of Physical Education, Faculty of Human Sciences, Hamedan Branch, Islamic Azad University, Hamadan, Iran.

Phone: +98 (918) 8156787

E-mail: khodaveisi_hamideh@yahoo.com
} 


\section{Highlights}

- The high activity of some muscles in people with low back pain causes muscle strain and fatigue.

- After using the lumbar back brace, the activity of the erector spinae and the rectus abdominis muscles was reduced during the mid-stance phase of walking.

\section{Plain Language Summary}

Regarding the high prevalence of low back pain, our study aimed to evaluate the effects of 8-week lumbar brace use on the amount of muscle activity in women with low back pain during walking. The activity of some muscles decreased after using 8 weeks of lumbar braces.

\section{Introduction}

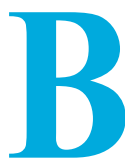

ack pain as a musculoskeletal disorder has an incidence rate of up to $80 \%$ worldwide, and anyone has experienced it once. Almost $90-95 \%$ of those first experiencing back pain recover in two months [1]. The reason for $85 \%$ of all back pains is unclear [2]. However, different reasons related to damage in organs such as bones, Faust joint, inter-ring discs, ligaments, nerves and muscles have been suggested in this regard [3].

Back pain is gender-dependent with more vulnerability in women [4]. Most researchers have suggested changes in the pattern and level of back and trunk area muscle activity for back pain. For example, Nelson Wong et al. (2012) indicated that those experiencing back pain while standing for a long time, compared with those without back pain, represent different muscle activity patterns in trunk extension movement (return from flexion). As a result, in the former group, erector spinae muscles are first activated, then gluteus maximus (top-down pattern) is activated, while in the latter group, the procedure was reverse (bottom-top pattern) [5, 6]. Arendt-Nilson stated that patients with chronic back pain have higher Electromyography (EMG) activity in erector spinae muscles in both sides of spines in the back area during the swing phase of walking. However, they found no significant difference in the activity of erector spinae muscles [7].

Hanada et al. (2011) reported that internal oblique and abdominal rectus muscles (anterior muscles) in patients with back pain are less active during walking compared to normal individuals. This is while trunk posterior area muscles (multifidus and erector spinae) in patients with back pain have more activity during walking [8]. In contrast, Van der Hulst et al. (2010) detected a greater muscle activity in rectus abdominis and erectus spinae muscles in patients with chronic back pain, during gain, compared with the normal subjects. Additionally, they reported no significant difference between the two groups in external oblique muscle activity [9].

Prior research suggested that increase in trunk muscle activity can result in spasm pain-brace pain cycle increases spinal loads by trunk muscle co-contraction [10]. Physicians claim that they can reduce some abnormal cases by increasing trunk activity muscles in therapeutic exercises in patients of back pain [11]. Different strategies are applied to treat chronic back pain. In this regard, some physicians use an external pelvic compression belt or braces to reduce the pain and increase muscle activity in different movements.

Different braces can be designed to stabilize the spine according to its symptoms. One of the prevalent braces used to treat back pain is lumbar support braces, designed to support erectus spinae muscles. Kim et al. surveyed the belt effect on the EMG activity of thigh and trunk muscles in women with back pain. They examined the subjects in face-up position and concluded that the left large dorsal and both erector spinae muscles activities and right gluteus maximus muscle in patients with back pain were greater than that of the normal group. Using a belt can reduce the activity of all these muscles in patients with back pain in extension movement and under the same condition [12].

In normal walking, coordinated patterns of trunk and trunk muscle activity maintain a dynamic balance to reduce energy costs and responding efficiently to motion deviations. Studies revealed that the coordination between trunk and pelvis movements in the frontal plane during walking is different in normal subjects [1]. This can be attributed to the guarded gait in patients with back 
pain. The movements mostly occur in the pelvis and the back area has less movement because of fear of pain. There is a paucity of information on the performance of trunk area muscles in individuals with chronic back pain. Moreover, the use of a back brace in individuals with chronic back pain, especially in walking has not been investigated. Therefore, the effects of using back brace for 8 weeks on the selected trunk muscles in walking among patients with chronic back pain remains unclear.

\section{Materials and Methods}

This was a quasi-experimental research with a pretest-post-test design and control group. After exact examinations, 22 subjects with chronic back pain were selected and placed into two experimental and control groups. The Ethics Committee of Hamadan Medical Science University approved this research and the obtained consent forms were confirmed by this committee. The formerly employed back pain for eight weeks. Before and after brace use, EMG activity was recorded in both groups.

The patients with back pain had a medium disability based on the Oswestry disability questionnaire. The research subjects reported no histories of surgery and ventricular system disorder. Additionally, abnormalities or disorders affecting the research results were diagnosed by a physician to exclude the affected subjects from the research. Other inclusion criteria were considered based on the previous studies. After the subjects referred to the laboratory and were prepared for the test, the hair on the examination area on the right side were cleaned with cotton and alcohol. Bipolar electrodes containing glue and conductor gel were used. The disposable surface electrodes in $30 \times 24 \mathrm{~mm}$ and an internal distance of $20 \mathrm{~mm}$ were installed on the selected muscles based on Surface EMG for Non-Invasive Assessment of Muscles (SENIAM) protocol [13].

EMG signals were collected from the surface EMG device of 16 channels (MYON model 320), with a sampling frequency of $1200 \mathrm{~Hz}$ and passed through a bypass filter of $10-450 \mathrm{~Hz}$. After the electrodes were placed, the subjects were requested to move on a direct path in a self-selected speed for $10 \mathrm{~m}$ to get ready for the main test $(3-5 \mathrm{~m})$.

For each group (before and after using back brace), three trials were recorded. The studied muscles consisted of erector spinae, external oblique, internal oblique, and abdominal rectus. Moreover, movement velocity was recorded by a chronometer. Studies revealed that MVIC tests increase acute pain in patients with back pain [12]. Therefore, submaximal contractions were used to normalize muscle activity. The reliability of the submaximal method in the activity of trunk muscles has been reported in patients with chronic back pain and normal subjects [14].

To investigate the voluntary submaximal contractions of erector spinae muscle, the subject lifted both knees to $5 \mathrm{~cm}$ above the testing table, with the knee at 90-degree flexion and maintained the position for 5 seconds. To record the submaximal isometric contractions in the rectus abdominis, abdominal internal oblique, and abdominal external oblique of the subjects in face-up supine position with the thigh joint at 45 degrees flexion and knee joint at 80 degrees flexion, the subject lifted legs about 1 $\mathrm{cm}$ for 5 seconds [15].

Subjects performed 3-5 second trials of SMVC, randomly for each muscle or group of muscles and were given a one-minute break between each trial. To detect the mid-stance, 6 cameras (Vicon T40-S) of $120 \mathrm{~Hz}$ frequency were employed. Markers were placed at the fifth metatarsus of right and left feet and the first metatarsus of right and left feet in the most protruded part of the left and right Achilles tendons. The mid-stance was considered as the time when the marker of left foot finger was lifted and the subject stood on the right foot. The back brace in this test was the sacro-lumbar support belt (Model: BB-OPPO2065, Taiwan).

To use the brace, first, in the sessions before and after eight weeks of using brace, the activity of the selected muscles was measured in walking. To assess the effect of 8 weeks of using brace, the subjects were requested to use brace in all activities for 8 weeks. To measure the perceived pain intensity, the Visual Analogue Scale (VAS) was used. The obtained data were processed using descriptive and inferential statistics. To investigate the normal distribution of data, the Shapiro-Wilk test was employed, along with the Independent samples ttest to analyze the data in SPSS. The significance level was set at $\mathrm{P} \leq 0.05$.

\section{Results}

Table 1 lists the demographic characteristics of the subjects. There was no significant difference between the 2 groups with regard to the age, height, weight, and BMI. The Independent Samples t-test results on the comparison of the activity of erector spine, external oblique, and internal oblique revealed no significant difference between the groups, before using the brace. In the control group, 
Table 1. Demographic characteristics of the subjects

\begin{tabular}{ccc}
\hline & & Mean \pm SD \\
\cline { 2 - 3 } Variable & Experimental & Control \\
\hline Age, $y$ & $44.7 \pm 3.7$ & $46.5 \pm 2.8$ \\
\hline Height, $\mathrm{cm}$ & $162.6 \pm 5.2$ & $164.6 \pm 4.1$ \\
\hline Weight, $\mathrm{kg}$ & $65.4 \pm 4.9$ & $63.4 \pm 5.2$ \\
\hline $\mathrm{BMI}, \mathrm{kg} / \mathrm{m}^{2}$ & $21.8 \pm 1.9$ & $20.5 \pm 1.6$ \\
\hline & & PHYSICAL TREA MENTS
\end{tabular}

Table 2. Comparing the modified RMS of muscles in the mid-stance phase of walking in the experimental group before and after using a brace

\begin{tabular}{ccccc}
\hline \multirow{2}{*}{ Muscles } & \multicolumn{2}{c}{ Mean \pm SD } & & P \\
\cline { 2 - 4 } & Pre-Test & Post-Test & t & $0.001^{*}$ \\
\hline Erector spinae & $65.3 \pm 24.7$ & $48.7 \pm 16.1$ & 4.447 & 0.110 \\
\hline External oblique & $50.9 \pm 28.7$ & $43.5 \pm 24.2$ & 1.708 & 0.107 \\
\hline Internal oblique & $41.5 \pm 20.2$ & $33.5 \pm 14.5$ & 1.725 & $0.041^{*}$ \\
\hline Abdominal rectus & $33.1 \pm 10.1$ & $20.3 \pm 8.5$ & 2.66 & \\
\hline
\end{tabular}

*Significance level $=0.05$

after 8 weeks, no difference was found in the pre-test and post-test in terms of the activity of erectus abdominis, external oblique, erector spinae and internal oblique.

Table 2 demonstrates the results of Paired t-test, before and after using a brace in the experimental group. After 8 weeks of using a brace, there was no significant difference in the activity of erector spinae, abdominal erectus, average EMG activity of external oblique and internal oblique before and after using a brace in the mid-stance phase of walking. Table 3 presents the Paired t-test results in respect to the subjects' pre-test and post-test pain scores in the experimental and control groups. A significant decrease in pain score was observed after using a brace, while no significant change was observed in the control group.

\section{Discussion}

The present research explored the effect of a back brace for 8 weeks on the EMG activity of trunk selected muscles in walking among the subjects with chronic back pain. After 3 months of using a brace, erector spinae muscle activity was lower than that of before using a brace.

Greater back muscles EMG activity in patients with chronic back pain in walking may be caused by difficulties in moving and pain [9]. Furthermore, increased trunk extensor may lead to spasm and greater pain. The higher activity of trunk extensor muscles may result in muscle fatigue due to muscle overuse which is likely to raise the load on the spine which is harmful [16]. EMG activity of erector spinae muscle is related to the load applied on the back inter-ring discs [17]. Therefore, the

Table 3. The Paired t-test results for the subjects' pre-test and post-test pain scores in the experimental and control groups

\begin{tabular}{|c|c|c|c|c|c|}
\hline \multirow{2}{*}{ Variable } & \multirow{2}{*}{ Group } & \multicolumn{2}{|c|}{ Mean $\pm S D$} & \multirow{2}{*}{$\mathbf{t}$} & \multirow{2}{*}{$\mathbf{P}$} \\
\hline & & Pre-Test & Post-Test & & \\
\hline & Experimental & $6 \pm 0.651$ & $3.11 \pm 0.66$ & 15.11 & $0.001 *$ \\
\hline \multicolumn{6}{|l|}{ Pain } \\
\hline & Control & $6.12 \pm 1.23$ & $5.63 \pm 1.24$ & 1.21 & 0.110 \\
\hline
\end{tabular}

*Significance level=0.05

PHYSICAL TREA $\$ MENTS 
greater activity level of erector spinae muscle may impose unfavorable forces to the spine [18].

Back brace application was effective in stabilizing back area in subjects with back pain due to a reduction of erector spinae muscle activity. Park et al. (2013) reported that pelvic belt used for imposing pressure on the selected area in normal subjects reduced the activity of abdominal muscles, including external oblique, internal oblique and abdominal rectus in the test of direct foot lift [19]. The tension and compression on ligaments of sacroiliac joints, using a belt in this area can evoke raffini and passini receptors, as the neural movement mechanism regulators of the joint dynamic control.

The simultaneous activity of these receptors provides an input source to an external pressure on the pelvis by the brace. Deformation of muscle receptors and sub-ligaments creates extra deep inputs [20]. Overall, the activity of these receptors provides physical sensual stimulations affecting the deep receptors of sacroiliac joints, kinetic neural control of adjacent muscles, and reduces muscle activity. However, the effect of brace on the activity of other upper and lower extremities and trunk must be investigated, along with other kinematic and kinetic variables, to explain brace biomechanical features. Using brace can decrease erector spinae muscle activity and abdominal erectus muscle in the mid-stance phase of walking. The muscle activity reduction can play an important role in pain reduction among the subjects with chronic back pain.

\section{Ethical Considerations}

\section{Compliance with ethical guidelines}

The Ethics Committee of Hamadan Medical Science University approved this research and the obtained consent forms were confirmed by this committee.

\section{Funding}

This project has been sponsored by Hamedan University of Medical Sciences.

\section{Authors contributions}

All authors contributed in preparing this article.

\section{Conflict of interest}

The authors declared no conflict of interest.

\section{References}

[1] Seay JF, Van Emmerik RE, Hamill J. Low back pain status affects pelvis-trunk coordination and variability during walking and running. Clinical Biomechanics. 2011; 26(6):572-8. [DOI:10.1016/j.clinbiomech.2010.11.012] [PMID]

[2] Waddell G, Burton AK. Occupational health guidelines for the management of low back pain at work: Evidence review. Occupational Medicine. 2001; 51(2):124-35. [DOI:10.1093/ occmed/51.2.124]

[3] Bird AR, Bendrups AP, Payne CB. The effect of foot wedging on electromyographic activity in the erector spinae and gluteus medius muscles during walking. Gait \& Posture. 2003; 18(2):81-91. [DOI:10.1016/S0966-6362(02)00199-6]

[4] Weksler N, Velan GJ, Semionov M, Gurevitch B, Klein M, Rozentsveig $\mathrm{V}$, et al. The role of sacroiliac joint dysfunction in the genesis of low back pain: The obvious is not always right. Archives of Orthopaedic and Trauma Surgery. 2007; 127(10):885-8. [DOI:10.1007/s00402-007-0420-x] [PMID]

[5] Nelson-Wong E, Alex B, Csepe D, Lancaster D, Callaghan JP. Altered muscle recruitment during extension from trunk flexion in low back pain developers. Clinical Biomechanics. 2012 27(10):994-8. [DOI:10.1016/j.clinbiomech.2012.07.007] [PMID]

[6] Wong TK, Lee RY. Effects of low back pain on the relationship between the movements of the lumbar spine and hip. Human Movement Science. 2004; 23(1):21-34. [DOI:10.1016/j. humov.2004.03.004] [PMID]

[7] Arendt-Nielsen L, Graven-Nielsen T, Svarrer H, Svensson P. The influence of low back pain on muscle activity and coordination during gait: A clinical and experimental study. Pain. 1996; 64(2):231-40. [DOI:10.1016/0304-3959(95)00115-8]

[8] Hanada EY, Johnson M, Hubley-Kozey C. A comparison of trunk muscle activation amplitudes during gait in older adults with and without chronic low back pain Physical Medicine and Rehabilitation. 2011; 3(10):920-8 [DOI:10.1016/j.pmrj.2011.06.002] [PMID]

[9] van der Hulst M, Vollenbroek-Hutten MM, Rietman JS, Hermens HJ. Lumbar and abdominal muscle activity during walking in subjects with chronic low back pain: Support of the "guarding" hypothesis. Journal of Electromyography and Kinesiology. 2010; 20(1):31-8. [DOI:10.1016/j.jelekin.2009.03.009] [PMID]

[10] Keir PJ, MacDonell CW. Muscle activity during patient transfers: A preliminary study on the influence of lift assists and experience. Ergonomics. 2004; 47(3):296-306. [DOI:10.10 80/0014013032000157922] [PMID]

[11] Fryer G, Morris T, Gibbons P. Paraspinal muscles and intervertebral dysfunction: Part one. Journal of Manipulative and Physiological Therapeutics. 2004; 27(4):267-74 [DOI:10.1016/j.jmpt.2004.04.008] [PMID]

[12] Kim JW, Kwon OY, Kim TH, An DH, Oh JS. Effects of external pelvic compression on trunk and hip muscle EMG activity during prone hip extension in females with chronic low back pain. Manual Therapy. 2014; 19(5):467-71. [DOI:10.1016/j.math.2014.04.016] [PMID]

[13] Hermens DH, Feriks B. Surface Electromyography for the Non-Invasive Assessment of Muscle (SENIAM). Brussels: European Union; 2005. 
[14] Dankaerts W, O'Sullivan PB, Burnett AF, Straker LM, Danneels LA. Reliability of EMG measurements for trunk muscles during maximal and sub-maximal voluntary isometric contractions in healthy controls and CLBP patients. Journal of Electromyography and Kinesiology. 2004; 14(3):333-42. [DOI:10.1016/j.jelekin.2003.07.001] [PMID]

[15] Allison GT, Godfrey P, Robinson G. EMG signal amplitude assessment during abdominal bracing and hollowing. Journal of Electromyography and Kinesiology. 1998; 8(1):51-7. [DOI:10.1016/S1050-6411(97)00004-7]

[16] Stokes IA, Gardner-Morse M. Lumbar spine maximum efforts and muscle recruitment patterns predicted by a model with multijoint muscles and joints with stiffness. Journal of Biomechanics. 1995; 28(2):177-86. [DOI:10.1016/00219290(94)E0040-A]

[17] Örtengren R, Andersson GB. Electromyographic studies of trunk muscles, with special reference to the functional anatomy of the lumbar spine. Spine. 1977; 2(1):44-52. [PMID]

[18] Cresswell AG, Oddsson L, Thorstensson A. The influence of sudden perturbations on trunk muscle activity and intraabdominal pressure while standing. Experimental Brain Research. 1994; 98(2):336-41. [DOI:10.1007/BF00228421] [PMID]

[19] Park KH, Ha SM, Kim SJ, Park KN, Kwon OY, Oh JS. Effects of the pelvic rotatory control method on abdominal muscle activity and the pelvic rotation during active straight leg raising. Manual Therapy. 2013; 18(3):220-4. [DOI:10.1016/j. math.2012.10.004] [PMID]

[20] Hogervorst T, Brand RA. Current concepts reviewmechanoreceptors in joint function. Journal of Bone and Joint Surgery. 1998; 80(9):1365-78. [DOI:10.2106/00004623199809000-00018] 